\title{
THE CENTRAL REGION OF THE PLANETARY NEBULA A58
}

\author{
D.L. POLLACCO and P.W. HILL \\ Department of Physics and Astronomy, North Haugh, St. Andrews, \\ Fife KY16 9SS, Scotland \\ and \\ R.E.S. CLEGG \\ Royal Greenwich Observatory, Madingley Road, Cambridge, CB3 OEZ, England
}

We present images and high-resolution spectra of the hydrogen deficient knot at the centre of the old planetary nebula A58. The spectra confirm that this region contains essentially no hydrogen, as previously suspected. Emission lines from the knot are broad (FWHM $\sim 180$ and $270 \mathrm{~km} / \mathrm{s}$ for [NII] and [OIII] lines respectively) and are blue-shifted by $\sim 100 \mathrm{~km} / \mathrm{s}$ relative to the systematic velocity.

We analyse these data in terms either of a one-sided collimated flow (the rear side flow being obscured or suppressed) or of dusty, spherical wind (where the rear side of the compact object is obscured by dust) with the data lending marginal support for the collimated flow model. The collimated flow or fast, $\mathrm{H}$-poor wind is very likely to be associated with the 1919 nova-like event in Aquila.

New spectra of the central star are also presented. 